\title{
HEDGING OF THE EUROPEAN OPTION IN DISCRETE TIME UNDER TRANSACTION COSTS DEPENDING ON TIME
}

Abstract. Hedging of the European option in a discrete time financial market with proportional transaction costs is considered. It is shown that for a certain class of options the set of portfolios which allow the seller to pay the claim of the buyer in quite a general discrete time market model is the same as the set of such portfolios under the assumption that the stock price movement is given by a suitable CRR model.

1. Introduction. In the paper we consider the problem of hedging the European option in a discrete time market model under proportional transaction costs. Typically in papers on hedging under proportional transaction costs the coefficients of the costs are constant over time. In this paper we allow these coefficients to vary.

The problem of hedging contingent claims in discrete time under proportional transaction costs was studied in many papers (see [1]-13]). However, it appears to be nontrivial to apply to the real market the results which were obtained for a general model (see [3], [4], [12]). From the computational point of view the so called Cox-Ross-Rubinstein model (CRR model) is very convenient since it easily yields the price of an option as well as the set of portfolios which allow hedging the option (see for instance [1], [2], [10], [11]). On the other hand the Cox-Ross-Rubinstein model seems to be too simple to be a proper description of the real stock price movement. However, if there are no transaction costs and the payoff function of the option is convex then the solution of the hedging problem in quite a general model of the stock price process is the same as in a proper binomial model. This fact

2010 Mathematics Subject Classification: Primary 91B24; Secondary 91G20.

Key words and phrases: European option, self-financing strategy, hedging, transaction costs. 
was proved for the market with one risky asset in [13] (see also [5] where the case of the European call option is considered) and in [9] for the case of an arbitrary number of assets. In [8] the model with proportional transaction costs is considered and a certain class of options is defined such that the set of portfolios which allow one to hedge an option in a model where changes of the stock price are bounded and i.i.d. random variables is the same as the set of such portfolios in a proper CRR model.

This paper contains the definition of another class of options such that the problem of finding the set of portfolios which enable hedging an option in quite a general model of stock price evolution simplifies to this problem in the CRR model. The convexity of suitable functions is an important part of this definition, and it is quite easy to verify if an option is an element of the class in question. The result therefore seems to be interesting for practitioners since it justifies the use of the CRR model to price derivatives for a certain class of options.

2. The model. Let $(\Omega, \mathcal{F}, P)$ be a probability space, $T$ a positive natural number and $\left\{\mathcal{F}_{t}, t=0, \ldots, T\right\}$ a family of $\sigma$-algebras such that $\mathcal{F}_{0}=$ $\{\emptyset, \Omega\}, \mathcal{F}_{t} \subseteq \mathcal{F}_{t+1}$ for $t=0, \ldots, T-1$ and $\mathcal{F}_{T}=\mathcal{F}$.We assume that $\Omega$ is finite (except Subsection 4.2).

Throughout this paper equalities and inequalities depending on $\omega \in \Omega$ hold for all $\omega \in \Omega$ if not stated otherwise.

We consider a market with two primary securities, a risky stock and a riskless bond, and assume that all assets are infinitely divisible.

The stock price movement is modeled by the process $\left\{S_{t}, t=0, \ldots, T\right\}$ where $S_{t}$ denotes the price of the stock at time $t$, for $t=0, \ldots, T$. We assume that $S_{t}$ is $\mathcal{F}_{t}$ measurable for $t=0, \ldots, T$.

In our model the stock price process satisfies the recursive formula

$$
S_{t+1}=\eta_{t+1} S_{t} \quad \text { for } t=0, \ldots, T-1
$$

where $S_{0}>0$ and $\left\{\eta_{t}\right\}_{t=1}^{T}$ is a sequence of i.i.d. random variables such that $\eta_{t} \in[d, u]$ and $0<d<u$.

We assume that

$$
P\left(\eta_{t}=e\right)>0 \quad \text { for } t=1, \ldots, T \text { and } e \in\{d, u\} .
$$

It is easily seen that the price of the stock is positive at all times.

We assume that $\mathcal{F}_{t}=\sigma\left(\eta_{u}, 1 \leq u \leq t\right)$ for $u=1, \ldots, T$.

In our model the bond earns interest with a nonnegative, constant rate $r$ satisfying the inequalities $d<1+r<u$.

In this model proportional transaction costs are paid when shares of the stock are traded. The cost of buying one share at time $t$ is $\left(1+\lambda_{t}\right) S_{t}$ where $\lambda_{t} \in[0, \infty)$ for $t=0, \ldots, T$, and the amount received for selling one share at time $t$ is $\left(1-\mu_{t}\right) S_{t}$ with $\mu_{t} \in[0,1)$ for $t=0, \ldots, T$. 
We assume that for every $t=0, \ldots, T-1$ the following inequalities hold:

$$
\begin{aligned}
& \left(1+\lambda_{t}\right)(1+r)-\left(1+\lambda_{t+1}\right) d \geq 0, \\
& \left(1+\lambda_{t+1}\right) u-\left(1+\lambda_{t}\right)(1+r) \geq 0, \\
& \left(1-\mu_{t+1}\right) u-\left(1-\mu_{t}\right)(1+r) \geq 0, \\
& \left(1-\mu_{t}\right)(1+r)-\left(1-\mu_{t+1}\right) d \geq 0 .
\end{aligned}
$$

For every $t=0, \ldots, T$ we define

$$
\rho_{t}(z)= \begin{cases}\left(1+\lambda_{t}\right) z & \text { if } z \geq 0 \\ \left(1-\mu_{t}\right) z & \text { if } z<0 .\end{cases}
$$

For all $\left(q_{1}, q_{2}\right) \in \mathbb{R}^{2}$ and $t=0, \ldots, T$ we define

$$
C_{\left(q_{1}, q_{2}\right)}^{t}=\left\{\left(v_{1}, v_{2}\right) \in \mathbb{R}^{2}: q_{1}-v_{1}+\rho_{t}\left(q_{2}-v_{2}\right) \leq 0\right\} .
$$

A trading strategy $(x, y)$ is a pair of processes $\left\{\left(x_{t}, y_{t}\right), t=0, \ldots, T-1\right\}$ where $x_{t}, y_{t}$ are $\mathcal{F}_{t}$ measurable for every $t=0, \ldots, T-1$. Here $x_{t}, y_{t}$ denote (cash) holdings of bonds and shares respectively by the seller at time $t$ (after transaction at that time). Moreover, for a strategy $(x, y)$ let $x_{-1}, y_{-1} \in \mathbb{R}$ denote the seller's initial holdings of bonds and shares respectively.

By convention we set $S_{-1}=S_{0}$.

A trading strategy $(x, y)$ is called self-financing if $x_{0}-x_{-1}+\rho_{0}\left(y_{0}-y_{-1}\right)$ $\leq 0$ and $x_{t}-(1+r) x_{t-1}+\rho_{t}\left(y_{t}-\eta_{t} y_{t-1}\right) \leq 0$ for $t=1, \ldots, T-1$. This means that at every trading time, the sales must finance possible purchases.

Denote by $\mathcal{A}$ the set of all self-financing trading strategies.

If $P\left(\eta_{t}=d\right)+P\left(\eta_{t}=u\right)=1$ for every $t=1, \ldots, T$ and $0<P\left(\eta_{t}=u\right)<1$ for every $t=1, \ldots, T$ then we have the so called Cox-Ross-Rubinstein model, denoted by $\operatorname{CRR}(u, d)$.

3. Some auxiliary results. Throughout this paper, if not stated otherwise, functions are defined on $(0, \infty)$, measurable, and take values in $\mathbb{R}$.

Let $p=\left(p_{1}, p_{2}\right)$ be a given pair of functions.

For every $t=0, \ldots, T$ we define

$$
c_{p, 1}^{t}(s)=\frac{p_{1}(s)}{1+\lambda_{t}}+p_{2}(s) \quad \text { and } \quad c_{p, 2}^{t}(s)=\frac{p_{1}(s)}{1-\mu_{t}}+p_{2}(s)
$$

for $s \in(0, \infty)$.

Throughout this paper, if not stated otherwise, equalities and inequalities depending on $s \in(0, \infty)$ hold for all $s \in(0, \infty)$.

For simplicity of notation set $\gamma_{t}=\left(1+\lambda_{t}\right) u-\left(1-\mu_{t}\right) d$ for $t=0, \ldots, T$. It is easily seen that $\gamma_{t}>0$ for $t=0, \ldots, T$. 
For given $p=\left(p_{1}, p_{2}\right)$ and $t=0, \ldots, T-1$ we define functions

$$
\begin{aligned}
& I_{p}^{1, t}(s)=\left(\frac{p_{2}(u s)}{u}-\frac{p_{2}(d s)}{d}\right)-\frac{1}{\left(1+\lambda_{t+1}\right) u}\left(p_{1}(d s)-p_{1}(u s)\right), \\
& I_{p}^{2, t}(s)=\frac{1}{\left(1-\mu_{t+1}\right) d}\left(p_{1}(d s)-p_{1}(u s)\right)-\left(\frac{p_{2}(u s)}{u}-\frac{p_{2}(d s)}{d}\right) .
\end{aligned}
$$

Let $\Psi$ denote the set of all pairs of functions $p$ such that

1. $c_{p, 1}^{T}, c_{p, 2}^{T}$ are convex,

2. $I_{p}^{1, T-1}(s) \geq 0$ and $I_{p}^{2, T-1}(s) \geq 0$ for all $s \in(0, \infty)$.

Definition 3.1. Let $\left(p_{1}, p_{2}\right)$ be a given pair of functions. By backward induction we define pairs of functions $p^{(t)}=\left(p_{1}^{(t)}, p_{2}^{(t)}\right)$ for $t=0, \ldots, T$ as follows:

$$
\begin{aligned}
p_{1}^{(T)}(s) & =p_{1}(s), \quad p_{2}^{(T)}=p_{2}(s), \\
p_{1}^{(t)}(s) & =\frac{\left(1-\mu_{t+1}\right)\left(1+\lambda_{t+1}\right)}{(1+r) \gamma_{t+1}}\left(u c_{p^{(t+1), 2}}^{t+1}(d s)-d c_{p^{(t+1), 1}}^{t+1}(u s)\right), \\
p_{2}^{(t)}(s) & =\frac{-(1+r)}{\left(1+\lambda_{t+1}\right) u} p_{1}^{(t)}(s)+\frac{1}{u} c_{p^{(t+1), 1}}^{t+1}(u s) .
\end{aligned}
$$

LEMMA 3.2. Let $\left(p_{1}, p_{2}\right)$ be a pair of functions. For all $s \in(0, \infty)$ and $t=0, \ldots, T-1$ the following identities hold:

$$
\begin{aligned}
& p_{1}^{(t+1)}(d s)-(1+r) p_{1}^{(t)}(s)+\rho_{t+1}\left(p_{2}^{(t+1)}(d s)-d p_{2}^{(t)}(s)\right)=0, \\
& p_{1}^{(t+1)}(u s)-(1+r) p_{1}^{(t)}(s)+\rho_{t+1}\left(p_{2}^{(t+1)}(u s)-u p_{2}^{(t)}(s)\right)=0 .
\end{aligned}
$$

For all $\theta \in[d, u], t=0, \ldots, T-1$ and a given pair of functions $p$ we define functions

$$
\begin{aligned}
L_{p}^{1, t, \theta}(s)= & \frac{(u-\theta)\left(1-\mu_{t+1}\right)}{\gamma_{t+1}} c_{p, 2}^{t+1}(d s) \\
& +\frac{\left(1+\lambda_{t+1}\right) \theta-\left(1-\mu_{t+1}\right) d}{\gamma_{t+1}} c_{p, 1}^{t+1}(u s)-c_{p, 1}^{t+1}(\theta s)
\end{aligned}
$$

and

$$
\begin{aligned}
L_{p}^{2, t, \theta}(s)= & \frac{\left(1+\lambda_{t+1}\right) u-\left(1-\mu_{t+1}\right) \theta}{\gamma_{t+1}} c_{p, 2}^{t+1}(d s) \\
& +\frac{(\theta-d)\left(1+\lambda_{t+1}\right)}{\gamma_{t+1}} c_{p, 1}^{t+1}(u s)-c_{p, 2}^{t+1}(\theta s) .
\end{aligned}
$$

For all $\omega \in \Omega, \theta \in[d, u], t=0, \ldots, T-1$ and a given pair of functions $p$ let

$$
\begin{aligned}
G_{p}^{\theta}(t)=\left\{(x, y) \in \mathbb{R}^{2}: y \geq \max \left\{\frac{-(1+r)}{\left(1+\lambda_{t+1}\right) \theta} x+\frac{1}{\theta} c_{p, 1}^{t+1}\left(\theta S_{t}\right),\right.\right. \\
\left.\left.\frac{-(1+r)}{\left(1-\mu_{t+1}\right) \theta} x+\frac{1}{\theta} c_{p, 2}^{t+1}\left(\theta S_{t}\right)\right\}\right\} .
\end{aligned}
$$


Moreover, let $\widehat{G}_{p}(t)=G_{p}^{d}(t) \cap G_{p}^{u}(t)$ for $t=0, \ldots, T-1$. It is clear that the set $G_{p}^{\theta}(t)$ depends on $\omega \in \Omega$ and consequently so does $\widehat{G}_{p}(t)$.

Lemma 3.3. For all $\omega \in \Omega, \theta \in[d, u]$ and $t=0, \ldots, T-1$ we have the following equivalence: $\widehat{G}_{p}(t)(\omega) \subseteq G_{p}^{\theta}(t)(\omega)$ if and only if $L_{p}^{1, t, \theta}\left(S_{t}(\omega)\right) \geq 0$ and $L_{p}^{2, t, \theta}\left(S_{t}(\omega)\right) \geq 0$.

Proof. We fix $\omega \in \Omega, \theta \in[d, u]$ and $t \in\{0, \ldots, T-1\}$, and omit $\omega$ in the notation. Let

$$
\begin{aligned}
& E_{1}^{\theta}=\left\{\left(v_{1}, v_{2}\right) \in \mathbb{R}^{2}: v_{2} \geq \frac{-(1+r)}{\left(1+\lambda_{t+1}\right) \theta} v_{1}+\frac{1}{\theta} c_{p, 1}^{t+1}\left(\theta S_{t}\right)\right\}, \\
& E_{2}^{\theta}=\left\{\left(v_{1}, v_{2}\right) \in \mathbb{R}^{2}: v_{2} \geq \frac{-(1+r)}{\left(1-\mu_{t+1}\right) \theta} v_{1}+\frac{1}{\theta} c_{p, 2}^{t+1}\left(\theta S_{t}\right)\right\} .
\end{aligned}
$$

It is easily seen that $\widehat{G}_{p}(t) \subseteq G_{p}^{\theta}(t)$ if and only if $p^{(t)}\left(S_{t}\right) \in E_{1}^{\theta} \cap E_{2}^{\theta}$.

By direct calculation we find that $p^{(t)}\left(S_{t}\right) \in E_{1}^{\theta}$ if and only if $L_{p}^{1, t, \theta}\left(S_{t}\right)$ $\geq 0$, and $p^{(t)}\left(S_{t}\right) \in E_{2}^{\theta}$ if and only if $L_{p}^{2, t, \theta}\left(S_{t}\right) \geq 0$.

By a standard calculation we obtain:

Lemma 3.4. For every $t=0, \ldots, T-1$ the following identities hold:

$$
\begin{aligned}
c_{p^{(t)}, 1}^{t}(s)= & \frac{\left(1+\lambda_{t+1}\right)\left(\left(1+\lambda_{t}\right)(1+r)-\left(1-\mu_{t+1}\right) d\right)}{\left(1+\lambda_{t}\right)(1+r) \gamma_{t+1}} c_{p^{(t+1), 1}}^{t+1}(u s) \\
& +\frac{\left(1-\mu_{t+1}\right)\left(\left(1+\lambda_{t+1}\right) u-\left(1+\lambda_{t}\right)(1+r)\right)}{\left(1+\lambda_{t}\right)(1+r) \gamma_{t+1}} c_{p^{(t+1), 2}}^{t+1}(d s)
\end{aligned}
$$

and

$$
\begin{aligned}
c_{p^{(t)}, 2}^{t}(s)= & \frac{\left(1+\lambda_{t+1}\right)\left(\left(1-\mu_{t}\right)(1+r)-\left(1-\mu_{t+1}\right) d\right)}{\left(1-\mu_{t}\right)(1+r) \gamma_{t+1}} c_{p^{(t+1), 1}}^{t+1}(u s) \\
& +\frac{\left(1-\mu_{t+1}\right)\left(\left(1+\lambda_{t+1}\right) u-\left(1-\mu_{t}\right)(1+r)\right)}{\left(1-\mu_{t}\right)(1+r) \gamma_{t+1}} c_{p^{(t+1), 2}}^{t+1}(d s) .
\end{aligned}
$$

Lemma 3.5. Let $p \in \Psi$. Then $I_{p^{(t+1)}}^{1, t}(s) \geq 0$ and $I_{p^{(t+1)}}^{2, t}(s) \geq 0$ for every $t=0, \ldots, T-1$.

Proof. By a straightforward calculation we get

$$
\begin{aligned}
& p_{1}^{(t)}(s)=\frac{\left(1-\mu_{t+1}\right)\left(1+\lambda_{t+1}\right) d u}{\gamma_{t+1}(1+r)} I_{p^{(t+1)}}^{2, t}(s)+\frac{p_{1}^{(t+1)}(u s)}{1+r}, \\
& p_{2}^{(t)}(s)=\frac{\left(1+\lambda_{t+1}\right) u}{\gamma_{t+1}} I_{p^{(t+1)}}^{1, t}(s)+\frac{1}{d} p_{2}^{(t+1)}(d s)
\end{aligned}
$$




$$
\begin{aligned}
& p_{1}^{(t)}(s)=\frac{p_{1}^{(t+1)}(d s)}{1+r}-\frac{\left(1-\mu_{t+1}\right)\left(1+\lambda_{t+1}\right) d u}{\gamma_{t+1}(1+r)} I_{p^{(t+1)}}^{1, t}(s), \\
& p_{2}^{(t)}(s)=\frac{1}{u} p_{2}^{(t+1)}(u s)-\frac{\left(1-\mu_{t+1}\right) d}{\gamma_{t+1}} I_{p^{(t+1)}}^{2, t}(s),
\end{aligned}
$$

for every $t=0, \ldots, T-1$.

We now use backward induction. Since $p \in \Psi$ we have $I_{p^{(T)}}^{1, T-1}(s) \geq 0$ and $I_{p^{(T)}}^{2, T-1}(s) \geq 0$. Assume that $I_{p^{(t+1)}}^{1, t}(s) \geq 0$ and $I_{p^{(t+1)}}^{2, t}(s) \geq 0$ for some $t \in\{1, \ldots, T-1\}$. From (3.1) and (3.3) we have

$$
p_{1}^{(t)}(d s)-p_{1}^{(t)}(u s)=\frac{\left(1-\mu_{t+1}\right)\left(1+\lambda_{t+1}\right) d u}{(1+r) \gamma_{t+1}}\left(I_{p^{(t+1)}}^{1, t}(u s)+I_{p^{(t+1)}}^{2, t}(d s)\right) .
$$

From (3.2) and (3.4) we have

$$
\frac{p_{2}^{(t)}(u s)}{u}-\frac{p_{2}^{(t)}(d s)}{d}=\frac{1+\lambda_{t+1}}{\gamma_{t+1}} I_{p^{(t+1)}}^{1, t}(u s)+\frac{1-\mu_{t+1}}{\gamma_{t+1}} I_{p^{(t+1)}}^{2, t}(d s) .
$$

By (3.5) and (3.6) we obtain

$$
\begin{aligned}
I_{p^{(t)}}^{1, t-1}(s)= & \frac{\left(1+\lambda_{t+1}\right)\left(\left(1+\lambda_{t}\right)(1+r)-\left(1-\mu_{t+1}\right) d\right)}{\left(1+\lambda_{t}\right)(1+r) \gamma_{t+1}} I_{p^{(t+1)}}^{1, t}(u s) \\
& +\frac{\left(1-\mu_{t+1}\right)\left(\left(1+\lambda_{t}\right)(1+r)-\left(1+\lambda_{t+1}\right) d\right)}{\left(1+\lambda_{t}\right)(1+r) \gamma_{t+1}} I_{p^{(t+1)}}^{2, t}(d s), \\
I_{p^{(t)}}^{2, t-1}(s)= & \frac{\left(1+\lambda_{t+1}\right)\left(\left(1-\mu_{t+1}\right) u-\left(1-\mu_{t}\right)(1+r)\right)}{\left(1-\mu_{t}\right)(1+r) \gamma_{t+1}} I_{p^{(t+1)}}^{1, t}(u s) \\
& +\frac{\left(1-\mu_{t+1}\right)\left(\left(1+\lambda_{t+1}\right) u-\left(1-\mu_{t}\right)(1+r)\right)}{\left(1-\mu_{t}\right)(1+r) \gamma_{t+1}} I_{p^{(t+1)}}^{2, t}(d s)
\end{aligned}
$$

and therefore by $(2.2)$ and $(2.4)$ we get $I_{p^{(t)}}^{1, t-1}(s) \geq 0$ and $I_{p^{(t)}}^{2, t-1}(s) \geq 0$.

As a consequence of (3.2), (3.4) and Lemma 3.5 we have:

LEMmA 3.6. Let $p=\left(p_{1}, p_{2}\right) \in \Psi$. Then $p_{2}^{(t+1)}(d s) \leq d p_{2}^{(t)}(s)$ and $p_{2}^{(t+1)}(u s) \geq u p_{2}^{(t)}(s)$ for all $s \in(0, \infty)$ and $t=0, \ldots, T-1$.

By a straightforward calculation we get

Lemma 3.7. For any pair of functions $p=\left(p_{1}, p_{2}\right)$, all $s \in(0, \infty)$ and $t=0, \ldots, T-1$ the following identities hold:

$$
\begin{array}{ll}
L_{p}^{1, t, d}(s)=\frac{\left(\lambda_{t+1}+\mu_{t+1}\right) d u}{\gamma_{t+1}} I_{p}^{1, t}(s), & L_{p}^{1, t, u}(s)=0, \\
L_{p}^{2, t, u}(s)=\frac{\left(\lambda_{t+1}+\mu_{t+1}\right) d u}{\gamma_{t+1}} I_{p}^{2, t}(s), & L_{p}^{2, t, d}(s)=0 .
\end{array}
$$

Concluding our technical results we obtain: 
THEOREM 3.8. Let $p \in \Psi$. Then $L_{p^{(t+1)}}^{1, t, \theta}(s) \geq 0$ and $L_{p^{(t+1)}}^{2, t, \theta}(s) \geq 0$ for all $\theta \in[d, u], s \in(0, \infty)$ and $t=0, \ldots, T-1$.

Proof. For all $s \in(0, \infty)$ and $t=0, \ldots, T-1$ let $h_{t}^{1, s}$ and $h_{t}^{2, s}$ be measurable functions defined on $[d, u]$ as follows:

$$
h_{t}^{1, s}(\theta)=L_{p^{(t+1)}}^{1, t, \theta}(s) \quad \text { and } \quad h_{t}^{2, s}(\theta)=L_{p^{(t+1)}}^{2, t, \theta}(s) \quad \text { for all } \theta \in[d, u] .
$$

By (2.3), (2.5) and Lemma 3.4 it is easily seen that $h_{t}^{1, s}$ and $h_{t}^{2, s}$ are concave for all $s \in(0, \infty)$ and $t=0, \ldots, T-1$. Therefore, we only need to show that $h_{t}^{1, s}(e) \geq 0$ and $h_{t}^{2, s}(e) \geq 0$ for all $s \in(0, \infty), e \in\{d, u\}$ and $t=0, \ldots, T-1$.

Indeed, from Lemma 3.7 we have $h_{t}^{1, s}(u)=0$ and $h_{t}^{2, s}(d)=0$ for all $s \in(0, \infty)$ and $t=0, \ldots, T-1$, while from Lemmas 3.5 and 3.7 we get $h_{t}^{1, s}(d) \geq 0$ and $h_{t}^{2, s}(u) \geq 0$ for all $s \in(0, \infty)$ and $t=0, \ldots, T-1$.

4. Hedging of the option. Let $\varphi=\left(\varphi_{1}, \varphi_{2}\right)$ be a given pair of functions. Define an option by a pair $\left(\varphi_{1}\left(S_{T}\right), \varphi_{2}\left(S_{T}\right)\right)$ of random variables where $\varphi_{1}\left(S_{T}\right), \varphi_{2}\left(S_{T}\right)$ denote the amounts (in cash) of bonds and shares respectively that are paid at time $T$ to the option's holder assuming that he exercises his claim. Throughout the paper we identify an option with the pay-off pair of functions $\varphi$.

An option will also be called a contingent claim or a European option since the option's holder can get his payment only at time $T$.

It can be easily seen that for each option $\varphi$ there exists a unique pair of functions $f_{\varphi}=\left(f_{\varphi, 1}, f_{\varphi, 1}\right)$ such that

$$
C_{\left(f_{\varphi, 1}(s), f_{\varphi, 2}(s)\right)}^{T}=C_{(0,0)}^{T} \cap C_{\left(\varphi_{1}(s), \varphi_{2}(s)\right)}^{T} \quad \text { for all } s \in(0, \infty) .
$$

We say that a trading strategy $(x, y) \in \mathcal{A}$ hedges the option $\varphi=\left(\varphi_{1}, \varphi_{2}\right)$ if

$$
\varphi_{1}\left(S_{T}\right)-(1+r) x_{T-1}+\rho_{T}\left(\varphi_{2}\left(S_{T}\right)-\eta_{T} y_{T-1}\right) \leq 0
$$

and

$$
\rho\left(\eta_{T} y_{T-1}\right) \leq(1+r) x_{T-1} .
$$

The condition (4.2) implies that the seller can pay the claim of the buyer, and from (4.3) it follows that the seller can reach simultaneously 0 in the number of bonds and shares. In other words (4.3) means that at time $T$ the seller can pay all his debts.

By (4.1) it is easily seen that (4.2) and (4.3) together are equivalent to the following inequality:

$$
f_{\varphi, 1}\left(S_{T}\right)-(1+r) x_{T-1}+\rho_{T}\left(f_{\varphi, 2}\left(S_{T}\right)-\eta_{T} y_{T-1}\right) \leq 0
$$

In other words a trading strategy $(x, y) \in \mathcal{A}$ hedges $\varphi$ if and only if it satisfies (4.4). 
The inequality (4.4) implies that the strategy $(x, y) \in \mathcal{A}$ hedges $\varphi$ if and only if it ensures the payments $f_{\varphi, 1}\left(S_{T}\right), f_{\varphi, 2}\left(S_{T}\right)$ in bonds and shares respectively at time $T$.

Let $\widetilde{x}_{t}=(1+r) x_{t-1}$ and $\widetilde{y}_{t}=\eta_{t} y_{t-1}$ for $t=0, \ldots, T-1$. For all $\omega \in \Omega$ and $t=0, \ldots, T-1$ define

$H_{\varphi}(t)(\omega)=\left\{v \in \mathbb{R}^{2}:\right.$ there exists $(x, y) \in \mathcal{A}$ such that $\left(\widetilde{x}_{t}, \widetilde{y}_{t}\right)(\omega)=v$ and $\left.P\left(f_{\varphi, 1}\left(S_{T}\right)-\widetilde{x}_{T}+\rho_{T}\left(f_{\varphi, 1}\left(S_{T}\right)-\widetilde{y}_{T}\right) \leq \widetilde{x}_{T} \mid \mathcal{F}_{t}\right)(\omega)=1\right\}$, $\widehat{H}_{\varphi}(t)(\omega)=\left\{v \in \mathbb{R}^{2}:\right.$ there exists $(x, y) \in \mathcal{A}$ such that $\left(x_{t}, y_{t}\right)(\omega)=v$ and $\left.P\left(f_{\varphi, 1}\left(S_{T}\right)-\widetilde{x}_{T}+\rho_{T}\left(f_{\varphi, 1}\left(S_{T}\right)-\widetilde{y}_{T}\right) \leq \widetilde{x}_{T} \mid \mathcal{F}_{t}\right)(\omega)=1\right\}$.

$H_{\varphi}(t)$ is the set of all pre-transaction portfolios which at time $t$ guarantee hedging of $\varphi$ at time $T$, and $\widehat{H}_{\varphi}(t)$ is the analogous set of all post-transaction portfolios.

Moreover, let $H_{\varphi}(T)=C_{\left(f_{\varphi, 1}\left(S_{T}\right), f_{\varphi, 2}\left(S_{T}\right)\right)}^{T}$. It is clear that $H_{\varphi}(T)$ is the set of all pre-transaction portfolios which at time $T$ guarantee the payments $f_{\varphi, 1}\left(S_{T}\right), f_{\varphi, 2}\left(S_{T}\right)$ in bonds and shares respectively.

For every $t=0, \ldots, T-1$ let $H_{\varphi}^{\mathrm{CRR}}(t)$ and $\widehat{H}_{\varphi}^{\mathrm{CRR}}(t)$ be defined in the same way as $H_{\varphi}(t)$ and $\widehat{H}_{\varphi}(t)$ respectively, assuming additionally that $P\left(\eta_{u}=d\right)+P\left(\eta_{u}=u\right) \quad$ and $\quad 0<P\left(\eta_{u}=u\right)<1 \quad$ for $u=t+1, \ldots, T$. $H_{\varphi}^{\mathrm{CRR}}(t)$ and $\widehat{H}_{\varphi}^{\mathrm{CRR}}(t)$ are the sets of pre-transaction and post-transaction portfolios respectively, which at time $t$ guarantee hedging of $\varphi$ at time $T$ if the stock price dynamics from time $t$ on is the same as in the $\operatorname{CRR}(d, u)$ model.

The seller's price of a contingent claim $\varphi$ is defined by

$$
\pi(\varphi)=\inf \left\{x_{0}+\rho_{0}\left(y_{0}\right):(x, y) \in \mathcal{A} \text { and }(x, y) \text { hedges } \varphi\right\} .
$$

It is easily seen that $H_{\varphi}(0)$ does not depend on $\omega \in \Omega$ and that $\pi(\varphi)=$ $\inf \left\{x \in \mathbb{R}:(x, 0) \in H_{\varphi}(0)\right\}$.

For every $t=0, \ldots, T-1$ we have the following fact:

Lemma 4.1. Let $v_{1}, v_{2} \in \mathbb{R}$ and $\omega \in \Omega$. If $\left(v_{1}, v_{2}\right) \in H_{\varphi}(t)(\omega)$ and $\widehat{H}_{\varphi}(t)(\omega) \subseteq C_{\left(v_{1}, v_{2}\right)}^{t}$ then $H_{\varphi}(t)(\omega)=C_{\left(v_{1}, v_{2}\right)}^{t}$.

Proof. We fix $\omega \in \Omega$ and omit it in the notation.

Suppose $\left(v_{1}, v_{2}\right) \in H_{\varphi}(t)$ and $\widehat{H}_{\varphi}(t) \subseteq C_{\left(v_{1}, v_{2}\right)}^{t}$. It is easily seen that $C_{\left(v_{1}, v_{2}\right)}^{t} \subseteq H_{\varphi}(t)$. We only have to prove that $H_{\varphi}(t) \subseteq C_{\left(v_{1}, v_{2}\right)}^{t}$. Let $\left(v_{3}, v_{4}\right) \in$ $H_{\varphi}(t)$. Then there exists $\left(v_{5}, v_{6}\right) \in \widehat{H}_{\varphi}(t)$ such that

$$
v_{5}-v_{3}+\rho_{t}\left(v_{6}-v_{4}\right) \leq 0 .
$$

Since $\widehat{H}_{\varphi}(t) \subset C_{\left(v_{1}, v_{2}\right)}^{t}$ we have

$$
v_{1}-v_{5}+\rho_{t}\left(v_{2}-v_{6}\right) \leq 0 .
$$


The last two inequalities imply

$$
v_{1}-v_{3}+\rho_{t}\left(v_{2}-v_{6}\right)+\rho_{t}\left(v_{6}-v_{4}\right) \leq 0 .
$$

It is easily seen that

$$
\rho_{t}\left(v_{2}-v_{6}\right)+\rho_{t}\left(v_{6}-v_{4}\right) \geq \rho_{t}\left(v_{2}-v_{4}\right) .
$$

By the last two inequalities we get

$$
v_{1}-v_{3}+\rho_{t}\left(v_{2}-v_{4}\right) \leq 0 .
$$

Therefore $\left(v_{3}, v_{4}\right) \in C_{\left(v_{1}, v_{2}\right)}^{t}$ and so $H_{\varphi}(t) \subseteq C_{\left(v_{1}, v_{2}\right)}^{t}$.

For every $i=0, \ldots, T$ let $f_{\varphi}^{(i)}=\left(f_{\varphi, 1}^{(i)}, f_{\varphi, 2}^{(i)}\right)$ denote the pair of functions obtained from $f_{\varphi}$ according to Definition 3.1.

We have the following fact (compare also Theorem 1 in [11] where the model with constant coefficients of the transaction costs is considered):

THEOREM 4.2. Let $\varphi$ be an option such that $f_{\varphi} \in \Psi$. Then $H_{\varphi}^{\mathrm{CRR}}(t)=$ $C_{\left(f_{\varphi, 1}^{(t)}, f_{\varphi, 2}^{(t)}\right.}^{t}$ for every $t=0, \ldots, T-1$. Proof. We use backward induction. Clearly $H_{\varphi}^{\mathrm{CRR}}(T)=C_{\left(f_{\varphi, 1}^{(T)}\left(S_{T}\right), f_{\varphi, 2}^{(T)}\left(S_{T}\right)\right)}^{T}$
Assume that for some $t=0, \ldots, T-1$ we have

$$
H_{\varphi}^{\mathrm{CRR}}(t+1)=C_{\left(f_{\varphi, 1}^{(t+1)}\left(S_{t+1}\right), f_{\varphi, 2}^{(t+1)}\left(S_{t+1}\right)\right)} .
$$

From Lemma 3.2 we have

$$
\begin{gathered}
f_{\varphi, 1}^{(t+1)}\left(d S_{t}\right)-(1+r) f_{\varphi, 1}^{(t)}\left(S_{t}\right)+\rho_{t+1}\left(f_{\varphi, 2}^{(t+1)}\left(d S_{t}\right)-d f_{\varphi, 2}^{(t)}\left(S_{t}\right)\right)=0 \\
f_{\varphi, 1}^{(t+1)}\left(u S_{t}\right)-(1+r) f_{\varphi, 1}^{(t)}\left(S_{t}\right)+\rho_{t+1}\left(f_{\varphi, 2}^{(t+1)}\left(u S_{t}\right)-u f_{\varphi, 2}^{(t)}\left(S_{t}\right)\right)=0 .
\end{gathered}
$$

From now on, if not stated otherwise, we fix $\omega \in \Omega$ and omit it in the notation.

Let $\left(w_{1}, w_{2}\right) \in \widehat{H}_{\varphi}^{\mathrm{CRR}}(t)$. By definition we have

$$
\begin{aligned}
& f_{\varphi, 1}^{(t+1)}\left(d S_{t}\right)-(1+r) w_{1}+\rho_{t+1}\left(f_{\varphi, 2}^{(t+1)}\left(d S_{t}\right)-d w_{2}\right) \leq 0 \\
& f_{\varphi, 1}^{(t+1)}\left(u S_{t}\right)-(1+r) w_{1}+\rho_{t+1}\left(f_{\varphi, 2}^{(t+1)}\left(u S_{t}\right)-u w_{2}\right) \leq 0 .
\end{aligned}
$$

From (4.6) and (4.8) we obtain

$$
\begin{aligned}
\rho_{t+1}\left(f_{\varphi, 2}^{(t+1)}\left(u S_{t}\right)-u w_{2}\right)-\rho_{t+1}\left(f_{\varphi, 2}^{(t+1)}\left(u S_{t}\right)-u f_{\varphi, 2}^{(t)}\left(S_{t}\right)\right) & \\
& +(1+r) f_{\varphi, 1}^{(t)}\left(S_{t}\right) \leq(1+r) w_{1} .
\end{aligned}
$$

From (4.5) and (4.7) we have

$$
\begin{aligned}
\rho_{t+1}\left(f_{\varphi, 2}^{(t+1)}\left(d S_{t}\right)-d w_{2}\right)-\rho_{t+1}( & \left.f_{\varphi, 2}^{(t+1)}\left(d S_{t}\right)-d f_{\varphi, 2}^{(t)}\left(S_{t}\right)\right) \\
& +(1+r) f_{\varphi, 1}^{(t)}\left(S_{t}\right) \leq(1+r) w_{1} .
\end{aligned}
$$


We will prove now that

$$
f_{\varphi, 1}^{(t+1)}\left(S_{t}\right)-w_{1}+\rho_{t}\left(f_{\varphi, 2}^{(t)}\left(S_{t}\right)-w_{2}\right) \leq 0 .
$$

There are two cases:

First, suppose $w_{2} \leq f_{\varphi, 2}^{(t)}\left(S_{t}\right)$. Then by Lemma 3.6 we get

$$
\begin{aligned}
\rho_{t+1}\left(f_{\varphi, 2}^{(t+1)}\left(u S_{t}\right)-u w_{2}\right)-\rho_{t+1}\left(f_{\varphi, 2}^{(t+1)}\left(u S_{t}\right)-u f_{\varphi, 2}^{(t)}\left(u S_{t}\right)\right) \\
=\left(1+\lambda_{t+1}\right) u\left(f_{\varphi, 2}^{(t)}\left(S_{t}\right)-w_{2}\right) .
\end{aligned}
$$

Moreover, from the inequality $w_{2} \leq f_{\varphi, 2}^{(t)}\left(S_{t}\right)$ we have

$$
\rho_{t}\left(f_{\varphi, 2}^{(t)}\left(S_{t}\right)-w_{2}\right)=\left(1+\lambda_{t}\right)\left(f_{\varphi, 2}^{(t)}\left(S_{t}\right)-w_{2}\right) .
$$

Consequently, by (2.3), (4.9) we obtain (4.11).

Now suppose $w_{2} \geq f_{\varphi, 2}^{(t)}\left(S_{t}\right)$. Then by Lemma 3.6 we get

$$
\begin{aligned}
\rho_{t+1}\left(f_{\varphi, 2}^{(t+1)}\left(d S_{t}\right)-d w_{2}\right)-\rho_{t+1}\left(f_{\varphi, 2}^{(t+1)}\left(d S_{t}\right)-d f_{\varphi, 2}^{(t)}\left(u S_{t}\right)\right) \\
=\left(1-\mu_{t+1}\right) d\left(f_{\varphi, 2}^{(t)}\left(S_{t}\right)-w_{2}\right) .
\end{aligned}
$$

From the inequality $w_{2} \geq f_{\varphi, 2}^{(t)}\left(S_{t}\right)$ we have

$$
\rho_{t}\left(f_{\varphi, 2}^{(t)}\left(S_{t}\right)-w_{2}\right)=\left(1-\mu_{t}\right)\left(f_{\varphi, 2}^{(t)}\left(S_{t}\right)-w_{2}\right) .
$$

Consequently, by (2.5), (4.10) we obtain (4.11)

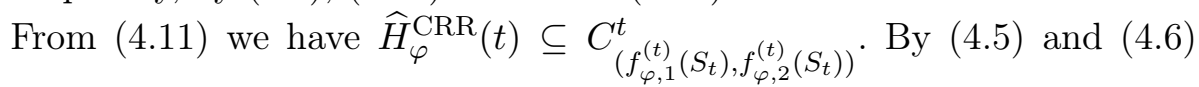
we obtain $\left(f_{\varphi, 1}^{(t)}\left(S_{t}\right), f_{\varphi, 2}^{(t)}\left(S_{t}\right)\right) \in H_{\varphi}^{\mathrm{CRR}}(t)$. Consequently, Lemma 4.1 implies that $H_{\varphi}(t)=C_{\left(f_{\varphi, 1}^{(t)}\left(S_{t}\right), f_{\varphi, 2}^{(t)}\left(S_{t}\right)\right)}^{\text {. }}$

The main result is:

THEOREM 4.3. Let $\varphi$ be an option such that $f_{\varphi} \in \Psi$. Then $H_{\varphi}(t)=$ $H_{\varphi}^{\mathrm{CRR}}(t)$ for every $t=0, \ldots, T-1$.

Proof. By Theorem 3.8 we have $L_{f_{\varphi}^{(t+1)}}^{1, t, \theta}\left(S_{t}\right) \geq 0$ and $L_{f_{\varphi}^{(t+1)}}^{2, t, \theta}\left(S_{t}\right) \geq 0$ for all $\omega \in \Omega, \theta \in[d, u]$ and $t=0, \ldots, T-1$. Thus from Lemma 3.3 we have $\widehat{G}_{f_{\varphi}^{(t+1)}}(t) \subseteq \bigcap_{\theta \in[d, u]} G_{f_{\varphi}^{(t+1)}}^{\theta}(t)$ for all $\omega \in \Omega$ and $t=0, \ldots, T-1$. Moreover, it is easy to verify that $f_{\varphi}^{(t)}\left(S_{t}\right) \in \widehat{G}_{f_{\varphi}^{(t+1)}}(t)$. Consequently,

$$
f_{\varphi}^{(t)}\left(S_{t}\right) \in \bigcap_{\theta \in[d, u]} G_{f_{\varphi}^{(t+1)}}^{\theta}(t) \quad \text { for all } \omega \in \Omega \text { and } t=0, \ldots, T-1 .
$$

We now use backward induction. 
It is clear that $H_{\varphi}(T)=C_{\left(f_{\varphi, 1}^{(T)}\left(S_{T}\right), f_{\varphi, 2}^{(T)}\left(S_{T}\right)\right)}^{T}$. Assume that for some $t=$ $0, \ldots, T-1$ we have

$$
H_{\varphi}(t+1)=C_{\left(f_{\varphi, 1}^{(t+1)}\left(S_{t+1}\right), f_{\varphi, 2}^{(t+1)}\left(S_{t+1}\right)\right)}^{t+}
$$

Then it is not difficult to check that $\bigcap_{\theta \in[d, u]} G_{f_{\varphi}^{(t+1)}}^{\theta}(t) \subseteq H_{\varphi}(t)$ for all $\omega \in \Omega$. Consequently, by (4.12) we get $f_{\varphi}^{(t)}\left(S_{t}\right) \in H_{\varphi}(t)$. Hence $C_{\left(f_{\varphi, 1}^{(t)}\left(S_{t}\right), f_{\varphi, 2}^{(t)}\left(S_{t}\right)\right)} \subseteq$ $H_{\varphi}(t)$ for all $\omega \in \Omega$. Thus by Theorem 4.2 we get $H_{\varphi}^{\mathrm{CRR}}(t) \subseteq H_{\varphi}(t)$ for all $\omega \in \Omega$. The reverse inclusion follows from (2.1).

REMARK 4.4. If we additionally assume that $r=0$ and $P\left(\eta_{t}=0\right)>0$ for every $t=0, \ldots, T$, then the set of pre-transaction portfolios that at a given time guarantee hedging of $\varphi$ such that $f_{\varphi} \in \Psi$, is the same as the analogous set for the American version of this option (pricing of the American option is considered e.g. in [7]).

\subsection{Examples}

4.1.1. The European call option with delivery. Throughout this example we assume that $\mu_{T}+\lambda_{T}>0$. The holder of the option has the right to buy one unit of the stock at the price $K$ at time $T$. We have $\varphi_{1}(s)=K$ and $\varphi_{2}(s)=s$. The pair $f_{\varphi}$ is given as follows:

$$
\begin{aligned}
& f_{\varphi, 1}(s)= \begin{cases}-K & \text { if } s \geq \frac{K}{1-\mu_{T}}, \\
\frac{1-\mu_{T}}{\lambda_{T}+\mu_{T}}\left(K-\left(1+\lambda_{T}\right)\right) & \text { if } \frac{K}{1+\lambda_{T}} \leq s<\frac{K}{1-\mu_{T}}, \\
0 & \text { otherwise },\end{cases} \\
& f_{\varphi, 2}(s)= \begin{cases}s & \text { if } s \geq \frac{K}{1-\mu_{T}}, \\
\frac{1+\lambda_{T}}{\lambda_{T}+\mu_{T}} s-\frac{K}{\lambda_{T}+\mu_{T}} & \text { if } \frac{K}{1+\lambda_{T}} \leq s<\frac{K}{1-\mu_{T}}, \\
0 & \text { otherwise. }\end{cases}
\end{aligned}
$$

We have

$$
c_{f_{\varphi}, 1}^{T}(s)=\left(s-\frac{K}{1+\lambda_{T}}\right)^{+} \quad \text { and } \quad c_{f_{\varphi}, 2}^{T}(s)=\left(s-\frac{K}{1-\mu_{T}}\right)^{+} .
$$

It is easily seen that the functions $c_{f_{\varphi}, 1}^{T}$ and $c_{f_{\varphi}, 2}^{T}$ are convex, and that $I_{f_{\varphi}}^{1, T-1}(s) \geq 0$ and $I_{f_{\varphi}}^{2, T-1}(s) \geq 0$. Thus $f_{\varphi} \in \Psi$. Consequently, $H_{\varphi}(t)=$ $H_{\varphi}^{\mathrm{CRR}}(t)$ for every $t=0, \ldots, T-1$ by Theorem 4.3 .

4.1.2. The European call option with cash settlement. Throughout this example we make the following assumption:

$$
\left(1-\mu_{T}\right) u \geq\left(1+\lambda_{T-1}\right)(1+r) .
$$


We have $\varphi_{1}(s)=(s-K)^{+}$and $\varphi_{2}(s)=0$. It is easily seen that $\varphi=f_{\varphi}$. By (4.13) we have $H_{\varphi}^{\mathrm{CRR}}(T-1)=C_{\left(g_{1}\left(S_{T-1}\right), g_{2}\left(S_{T-1}\right)\right)}^{T-1}$ where

$$
\begin{aligned}
& g_{1}(s)= \begin{cases}\frac{-K}{1+r} & \text { if } s \geq K / d, \\
\frac{(u s-K)}{(d-u)(1+r)} d & \text { if } K / u \leq s<K / d, \\
0 & \text { otherwise, }\end{cases} \\
& g_{2}(s)= \begin{cases}\frac{s}{1-\mu_{T}} & \text { if } s \geq K / d, \\
\frac{(u s-K)}{\left(1-\mu_{T}\right)(u-d)} & \text { if } K / u \leq s<K / d, \\
0 & \text { otherwise. }\end{cases}
\end{aligned}
$$

It is easy to verify that $H_{\varphi}(T-1)=H_{\varphi}^{\mathrm{CRR}}(T-1)$ and so $H_{\varphi}(T-1)=$ $C_{\left(g_{1}\left(S_{T-1}\right), g_{2}\left(S_{T-1}\right)\right.}^{T-1}$.

Let $g=\left(g_{1}, g_{2}\right)$. By $(2.4),(2.5)$ and (4.13) it is easy to check that the functions $c_{g, 1}^{T-1}$ and $c_{g, 2}^{T-1}$ are convex. By (2.4) we get $I_{g}^{1, T-2}(s) \geq 0$ and $I_{g}^{2, T-2}(s) \geq 0$. Therefore for the time horizon $T-1$ instead of $T$ we will have $g \in \Psi$.

Consequently, by Theorem 4.3 with the new terminal date $T-1$ we obtain $H_{\varphi}(t)=H_{\varphi}^{\mathrm{CRR}}(t)$ for every $t=0, \ldots, T-2$.

4.2. Hedging in a generalized model. In this subsection we assume a general $\Omega$ which does not have to be finite. Moreover, from now we assume that the stock price dynamics satisfies the following assumption weaker than (2.1).

Assumption 4.5. $P\left(\eta_{t+1}<d+\varepsilon\right)>0$ and $P\left(\eta_{t+1}>u-\varepsilon\right)>0$ for all $\varepsilon>0$ and $t=0, \ldots, T-1$.

For all $\varepsilon>0$ and $t=0, \ldots, T-1$ let $\Delta_{t}^{\varepsilon}$ denote the set of all sequences $\left(\delta_{n}\right)_{n=1}^{T-t}$ such that $0<\delta_{n}<\varepsilon$ for $n=1, \ldots, T-t$.

For all $\varepsilon>0, t=0, \ldots, T-1$ and $\delta \in \Delta_{t}^{\varepsilon}$ let $H_{\varphi}^{\varepsilon, \delta}(t)$ be defined in the same way as $H_{\varphi}(t)$ assuming in addition that $P\left(\eta_{t+1}=d+\delta_{v-t+1}\right)+P\left(\eta_{t+1}=\right.$ $\left.u-\delta_{v-t+1}\right)=1$ and $0<P\left(\eta_{t+1}=d-\delta_{v-t+1}\right)<1$ for $v=t, \ldots, T-1$.

For every $t=t, \ldots, T-1$ we have:

LEMMA 4.6. If $f_{\varphi, 1}$ and $f_{\varphi, 2}$ are continuous functions then $H_{\varphi}(t) \subseteq$ $H_{\varphi}^{\mathrm{CRR}}(t)$ for all $\omega \in \Omega$.

Proof. We fix $\omega \in \Omega$ and omit it in the notation. 
Assume that $\left(v_{1}, v_{2}\right) \in H_{\varphi}(t)$. By Assumption 4.5, for every $\varepsilon>0$ there exists a sequence $\delta \in \Delta_{t}^{\varepsilon}$ such that $\left(v_{1}, v_{2}\right) \in H_{\varphi}^{\varepsilon, \delta}(t)$. Since we can take $\varepsilon$ arbitrarily close to 0 and the functions $f_{\varphi, 1}$ and $f_{\varphi, 2}$ are continuous we get $\left(v_{1}, v_{2}\right) \in H_{\varphi}^{\mathrm{CRR}}(t)$.

In our generalized model, we have the following theorem which is similar to Theorem 4.2:

THEOREM 4.7. Let $\varphi$ be an option such that $f_{\varphi} \in \Psi$ and $f_{\varphi, 1}, f_{\varphi, 2}$ are continuous functions. Then $H_{\varphi}(t)=H_{\varphi}^{\mathrm{CRR}}(t)$ for all $\omega \in \Omega$ and $t=$ $0, \ldots, T-1$.

Proof. It is clear that $H_{\varphi}(T)=C_{\left(f_{\varphi, 1}^{(T)}\left(S_{T}\right), f_{\varphi, 1}^{(T)}\left(S_{T}\right)\right)}^{T}$. Assume that for some $t=1, \ldots, T-1$ we have

$$
H_{\varphi}(t+1)=C_{\left(f_{\varphi, 1}^{(t+1)}\left(S_{T+1}\right), f_{\varphi, 1}^{(t+1)}\left(S_{t+1}\right)\right)}^{t+1} .
$$

Following the lines of the proof of Theorem 4.3 we get $H_{\varphi}^{\mathrm{CRR}}(t) \subseteq H_{\varphi}(t)$ for all $\omega \in \Omega$, and Lemma 4.6 yields the reverse inclusion.

Acknowledgements. This research was supported by grant PBZ KBN no. 016/P03/99.

\section{References}

[1] B. Bensaid, J.-P. Lesne, H. Pagès and J. Scheinkman, Derivative asset pricing with transaction costs, Math. Finance 2 (1992), 63-86.

[2] P. P. Boyle and T. Vorst, Option replication in discrete time with transaction costs, J. Finance 47 (1992), 271-293.

[3] F. Delbaen, Y. M. Kabanov and E. Valkeila, Hedging under transaction costs in currency markets: a discrete-time model, Math. Finance 12 (2002), 45-61.

[4] Y. Kabanov, M. Rásonyi and Ch. Stricker, No arbitrage criteria for financial markets with efficient friction, Finance Stoch. 6 (2002), 371-382.

[5] D. E. Kashcheev, On the option pricing for a generalization of the binomial model, J. Math. Sci. (N.Y.) 99 (2000), 1267-1272.

[6] M. Kociński, Optimality of the replicating strategy for American options, Appl. Math. (Warsaw) 26 (1999), 93-105.

[7] -, Pricing of the American option in discrete time under proportional transaction costs, Math. Methods Oper. Res. 53 (2001), 67-88.

[8] - Hedging of the European option in discrete time under proportional transaction costs, ibid. 59 (2004), 315-328.

[9] M. Motoczyński and Ł. Stettner, On option pricing in the multidimensional CoxRoss-Rubinstein model, Appl. Math. (Warsaw) 25 (1998), 55-72.

[10] M. Rutkowski, Optimality of replication in the CRR model with proportional transaction costs, ibid. 25 (1998), 29-53.

[11] Ł. Stettner, Option pricing in the CRR model with proportional transaction costs: A cone transformation approach, ibid. 24 (1997), 475-514. 
[12] Ł. Stettner, Option pricing in discrete time incomplete market models, Math. Finance 10 (2000), 305-321.

[13] G. Tessitore and J. Zabczyk, Pricing options for multinomial models, Bull. Polish Acad. Sci. Math. 44 (1996), 363-380.

Marek Andrzej Kociński

Wydział Zastosowań Informatyki i Matematyki

Szkoła Główna Gospodarstwa Wiejskiego

Nowoursynowska 159

02-776 Warszawa, Poland

E-mail: marek_kocinski@sggw.pl

Received on 23.6.2004;

revised version on 11.10.2006 and 12.3.2010 